\title{
Editorial
}

\section{Determining Health Risks of New Hazards — We Can Do Better!}

Efficient prevention of industrial health problems is based on the availability of a human dose-response relationships and the factors that modify them. These can be used to estimate an individual's risk of adverse effects given an appropriate measure of his or her exposure intensity and its duration. Unfortunately, an individual's risk cannot be measured directly because risk is a property of groups of exposed individuals and must be determined by epidemiologic studies. As a result, when a new industry is developed around new materials, we usually cannot directly determine the health risks the materials may pose. Additionally, we cannot be reasonably certain of the human risks using data from animal, cell or other tests. Humans interact with environmental/occupational exposures in complex behavioral, biochemical, genetic and physiologic ways that are not well simulated in the lab. Society is also reluctant to limit the development of new materials, processes and ideas that may lead to important and exciting applications. One consequence of all of these limitations is a billion dollar industry can develop before enough human subjects have received enough exposure to do a useful epidemiologic study. If we better integrate multidisciplinary studies of new hazards, we can do better and quicker risk characterizations.

A diagram of the relationship between environmental and occupational exposure and the development of disease is shown in Fig. 1. A broad range of disciplines is needed to do a complete evaluation of these risk relationships, as shown along the bottom line of Fig. 1. However, the study of risks has become compartmentalized and disconnected. We liken what we are studying to an elephant. As in the Indian story of the six blind men and the elephant ${ }^{2)}$, each disciplinary group of scientists studies a small portion of the elephant with simplifying assumptions to make the problem tractable. The exposure and behavioral scientists, toxicologists, clinical scientists, and epidemiologists each argue the truth of their findings and may imply that the divergent findings of other disciplines are misleading or mistaken. Yet, each group has made important and relevant observations about aspects of occupational health problems. However, as the complexity has grown and exposure levels have decreased, single specialty studies have become less informative, and important opportunities for needed collaborations have been missed. In addition, there are risk assessment and policy experts who attempt to integrate the findings with further assumptions as they try to understand the "big picture" (the whole elephant). This integration is required to formulate efficient interventions, such as allowable exposures, to limit or eliminate hazards. I am proposing that this compartmentalized approach where specialized disciplinary groups work largely alone has left gaps about the nature of the hazards and risks. Some of the gaps associated with toxic chemical exposures are given below.

Exposure assessors do not include information on behavioral and sensory parameters that can modify contact with environmental contaminants, including exposure avoidance, temporal variation in exposure, and physical activity during exposure (breathing rate and mouth breathing).

Exposure studies have not included evaluation of sensory effects, such as irritation, strong odors, or other physiologic responses that might trigger exposure avoidance behavior.

Temporal exposure data (air and skin exposure intensities are highly variable) and physiological parameters

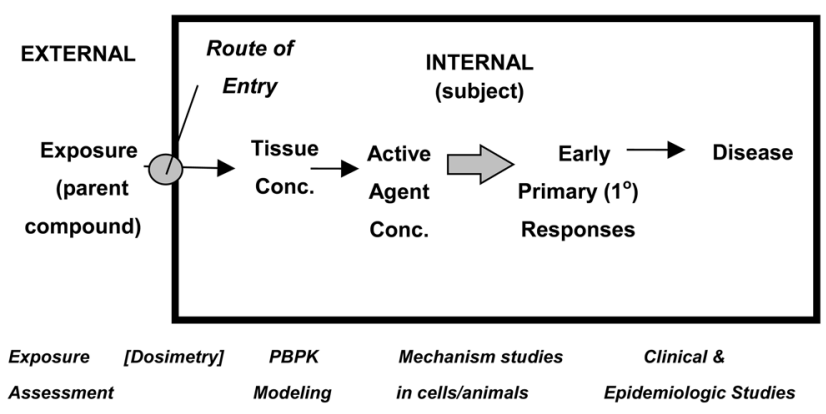

Fig. 1. Simplified diagram of the process relationships between exposure and disease. The relevant research specialties are shown in the box below the diagram ${ }^{1)}$. 
(breathing rate and cardiac output) are rarely measured for integration in internal dosimetry estimates, such as uptake, metabolism, genetics and their modifiers to provide more precise estimates of target tissue dose that define doseresponse relationships, and may define who has the highest risk.

Risk assessors use mechanistic descriptions of responses and metabolism parameters extrapolated from studies in animals, but rarely demonstrate that animal species responses do represent human risks.

Epidemiologists use only the crudest estimates or proxies for exposure in studies of human effects, which has long been recognized as a limitation of these types of studies.

Clinical data from suspected cases of hazardous exposures has not included reports of anything but limited comments on reported exposure conditions. There rarely has been follow up to obtain detailed on-site information on conditions of exposure. Likewise, clinical data is rarely used to indicate the time course of the responses and any recovery, if there is one.

The above list is not an exhaustive review of the points where useful collaborative data and research connections might be made between the research disciplines. The list reflects my experience with toxic chemical hazards. Ergonomic and psycho-social effects could be similarly evaluated.

My awareness of these issues was raised by my work on several governmental committees, which were examining the scientific literature and methods to determine if exposure to a particular agent was a hazard. As we reviewed the supporting scientific papers I noted the often repeated criticism of epidemiologic studies because they contain very limited exposure assessments. This is a major problem because observing an exposure-risk relationship is required to demonstrate causality ${ }^{3}$. Likewise, toxicological studies are criticized for using exposure conditions or materials that do not represent human exposures, or there are concerns that the test animals have biochemical or genetic differences from humans that make their responses noncomparable with humans. For example, benzene exposure in rats and mice does not cause leukemia, but benzene is a demonstrated cause of human leukemia ${ }^{4}$. Epidemiologic studies have also used biomarkers of exposure without adequate determination of factors that may modify their relationships, such as background levels of endogenous levels of the biomarker. Many of the exposure studies have not considered relevant psycho-social factors that will affect exposures and can add to misclassification.
For a variety of reasons, large integrated projects to study all of the dimensions defining an occupational hazard, as shown in Fig. 1, have not been conducted. Major limitations preventing this type of study have been limits on research funding, difficulty properly reviewing such multidisciplinary research applications, and institutional limits on organizing and leading such large studies. Some national occupational health institutes have organized collaborations that have successfully conducted large epidemiologic studies, such as industry-wide studies in Japan, China, USA, EU, and Scandinavia. However, some of these have not included concurrent detailed dosimetry studies of uptake by routes of entry. I believe that we now have the tools needed to allow us to study personal exposures and determine the responses to those exposures.

In depth studies can be conducted of modest groups of 50-100 individuals, where each subject can be evaluated in depth with repeated measures of exposure, internal biomarkers and health outcomes. Subgroups, with differences in age, race and gender, can provide considerable insight into genetic, epigenetic, metabolic and physiologic responses, and their variability within and between individuals. Some of these aspects can be studied by bringing selected groups of occupationally exposed individuals into the lab for controlled, permissible low-level exposures. Careful dosimetry measurements can be used to learn about uptake by route of entry, formation of metabolites and markers of cellular damage. Highly sensitive analytical techniques can measure trace substances in blood, urine and other body fluids. The data from these studies can provide the information needed for extrapolating risks. After the in-depth studies have identified the important parameters defining risk, large populations can be studied to look for distributions of parameters and infrequent high risk or susceptible subpopulations. This approach is much more efficient than trying to do highly detailed studies of large populations. Additionally when a new material is discovered, such as nano-materials, or production process is developed with unevaluated intermediates, smaller groups of workers may be evaluated to see if their exposures are producing troublesome effects before the new industrial activity has grown into a billion dollar industry. We can do better than waiting to see what happens.

\section{References}

1) National Research Council (USA) (1983) Committee on the Institutional Means for Assessment of Risks to Public Health. Risk assessment in the federal government: 
managing the process. National Academy Press, Washington, D.C.

2) Saxe JG (1995) Blind men and an elephant. In: Gardner M (Ed), 124, Famous Poems from Bygone Days: Courier Dover Publications, N. Chelmsford, MA.

3) Smith TJ, Kriebel D (2010) A biologic approach to environmental assessment and epidemiology. 425, Oxford
University Press, New York.

4) National Toxicology Program (USA) (1986) (Toxicology and Carcinogenesis Studies of Benzene (CAS No. 71-432) in F344/N Rats and B6C3F1 Mice (Gavage Studies). Technical Report No. 289. NIH Publication no. 86-2545. Research Triangle Park, NC, and Bethesda, MD.

Thomas J. SMITH Harvard School of Public Health, USA 